\title{
TEST DE OPERACIONES BÁSICAS PARA EL APRENDIZAJE DE LAS MATEMÁTICAS
}

\section{Luis A. Vicuña Peri}

\section{RESUMEN}

El propósito del presente estudio consiste en brindar a la comunidad psicológica y pedagógica un test que permite evaluar las operaciones básicas para el aprendizaje de las matemáticas y a su vez planificar las estrategias correctivas.

Se ha tomado en cuenta los aportes de Jean Piaget en lo concerniente al desarrollo de la función cognitiva integrada por el pensamiento lógico y la organización de la realidad,

El test se probó con una muestra del 1008 estudiantes que cursan el nivel secundario cuyas edades van desde los 12 hasta los 17 años con 11 meses, de colegios estatales de Lima,

Para la Confiabilidad se utilizó el método de las mitades, se encontró coeficientes $R$ de Spearman Brown de 0,81 hasta 0,94, siendo los índices de fiabilidad de 0,90 hasta 0,97. También se estimó la con fiabilidad por el método de la consistencia interna utilizando la ecuación de Kuder-Richardson, encontrando coeficientes de 0,86 hasta $\mathbf{0 , 9 0 .}$

La Validez de Constructo, confirmó que efectivamente los estudiantes con alto rendimiento en el test, tenían un rendimiento promedio en matemáticas significativamente mayor respecto a los estudiantes de bajo rendimiento en la prueba.

En la Validez Predictiva, que resulta correlacionando cada subtest y el puntaje total con el rendimiento en la asignatura de matemáticas se encontró coeficientes de 0,31 hasta 0,60.

Para la Validez Empírica, con el análisis correlacional intertest y test-total se encontró en todos los casos coeficientes de correlación muy significativos, pero en ningún caso el coeficiente es tal que indique que una variable mide lo mismo que la otra, manteniendo su independencia.

Mediante el Análisis Factorial se asume que los cinco factores son partes de un único factor denominado operaciones básicas para las matemáticas, lo que se confirmó mediante el test SCREE de Cattell, y el programa de Kaiser, así como el Ajuste de Bondad e índice de Adecuación realizado con el programa EQS, por lo que se demostró que el modelo ideal es igual al modelo teórico de un solo factor.

Palabras clave: Seriación, Inclusión, Interpretación numérica, clasificación, invariación.

\begin{abstract}
The purpose of the present study consists on offering to the psychological and pedagogic community a test that allows to evaluate the basic operations for the learning of the mathematics and in turn to plan the corrective strategies.

$\mathrm{He} / \mathrm{she}$ has taken into account the contributions of Jean Piaget in the concerning thing lo the development of the Cognitive integrated function by the logical thought and the organization of the reality.

The test was proven with a sample of the 1008 students that you/they study the secondary level whose ages go from the 12 to the 17 ages with 11 months, of slate schools of Lima.

For the Dependability the method of the halves was used, he/she was coefficients $R$ of Spearman Brown 0,81 up to 0,94, being the indexes of reliability of 0,90 up to 0,97. He/she was also considered the dependability for the method of the internal consistency using the equation of KuderRichardson, finding coefficients of 0,86 up to 0,90.

The Validity of construct validity, confirmed that indeed the students with high yield in the test, had a yield average in significantly more mathematics regarding the students of low yield in the test.

In the Predictive validity that is correlating each subtest and the total score with the yield in the subject of mathematics was coefficients of 0,31 up to 0,60,

For the Empiric Validity, with the analysis intertest correlation and total-test it was in all the cases very significant correlation coefficients, but in all case the coefficient is such that it indicates that a variable measures the same thing that the other one, maintaining its independence.

By means of the Factorial Analysis it is assumed that the five factors are parts of an only factor, denominated basic operations for the mathematics what you confirmed by means of the test SCREE of Cattell and the program of Kaiser, as well as the Adjustment of Kindness and Index of Adaptation carried out with the program EQS. for what was demonstrated that the ideal pattern is similar to the theoretical pattern of a single factor
\end{abstract}

Key Words: Seriation, Incorporation, numerical Interpretation, classification, unvariation. 
En nuestro país, como en muchos otros, la asignatura de matemáticas registra la mayor tasa de reprobados, como se puede comprobar revisando los informes anuales del rendimiento escolar dentro de los programas educativos regulares. Ante esta situación la pedagogía ha desarrollado recursos didácticos sin que el problema haya sido resuelto significativamente.

Para encontrar una adecuada respuesta a ello es necesario revisar los factores que influirían en el aprendizaje de las matemáticas. Por ello nos proponemos analizar dichos factores desde dos perspectivas, la del educador y la del alumno.

Respecto a la primera; el docente desarrollará la enseñanza de dos posibles formas, la colectiva y la individual.

En la enseñanza colectiva, mayoritaria en nuestro país, el profesor evalúa el logro de sus objetivos mediante el rendimiento de sus alumnos en los exámenes. El problema de utilizar este método radica en que no se evalúa el dominio de los puntos que encierra el curso, sino un porcentaje sesgado de contenidos que el docente estima pertinentes. De este porcentaje que el profesor evalúa, los estudiantes son promocionados con tan solo resolver el $51 \%$ de lo examinado, desempeño que no representa ni siquiera una proporción similar de los objetivos de la materia. Estos resultados son ilusos, puesto que el docente no alcanza a conocer si los alumnos han desarrollado las habilidades necesarias que el aprendizaje de las matemáticas permite.

En la enseñanza individualizada es evidente la disminución de los problemas en el aprendizaje de las matemáticas. El profesor, en estos casos está en la capacidad de descubrir las limitaciones de entendimiento del alumno, actuar sobre ellas y disminuirlas. Pero esta forma de enseñanza, si bien alcanza un nivel aceptable en la erradicación de dichos problemas, su utilización en nuestro medio es bastante restringida dado el alto costo que involucran. La gran virtud de la enseñanza individualizada se encuentra en el facilitar al profesor un conocimiento intuitivo respecto a las dificultades de su alumno.

Aún en este caso, la necesidad de proporcionar a la comunidad pedagógica de un instrumento que permita dar a conocer el nivel de repertorios básicos de los alumnos es indispensable. Dicho conocimiento permitirá al profesor la planificación de medidas preventivas y correctivas de las deficiencias en el aprendizaje de las matemáticas bajo el sustento de procesos intermedios, diferentes a la simple adquisición de reglas y principios del lenguaje matemático. Estos facilitarán al estudiante el entendimiento de los mismos, capacitándolos para su generalización y transferencia a nuevos aprendizajes.

Los procesos intermedios que consideramos indispensables, siguiendo los hallazgos de Jean Piaget corresponden a la naturaleza de la reversibilidad del pensamiento, que involucra las operaciones lógicas de inclusión, seriación y principios de la invariación de la sustancia, materia y energía. Junto a estos procesos consideramos también importante la capacidad desarrollada para transcribir y descifrar expresiones del lenguaje matemático.

Estos procesos intermedios deben haber alcanzado el nivel esperado para cada fase del desarrollo psíquico. Los mismos obedecen a las leyes del desarrollo y maduración, sin dejar de lado la estimulación apropiada en cada etapa. El conocimiento del nivel madurativo en el que se encuentra el estudiante nos permitirá aún en el caso de la enseñanza colectiva planificar y llevar a cabo las acciones pedagógicas que el caso amerite. Ello nos permitirá replantear las acciones pedagógicas en función al nivel alcanzado por los estudiantes, teniendo en cuenta tanto los casos de déficit como los de sobresaliente desempeño.

Para tal fin nos proponemos elaborar un instrumento evaluación válido y confiable capaz de detectar los problemas que podrían presentarse en el aprendizaje de las matemáticas teniendo en cuenta los procesos intermedios por ella requeridos cuyo aporte a 
la comunidad pedagógica y psicológica resulten realmente importantes, con el test que hemos denominado "Test de Operaciones básicas para el aprendizaje de las Matemáticas".

De forma que cuando trabajamos con una muestra de estudiantes que cursan el nivel secundario en colegios nacionales de Lima Metropolitana, queremos despejar las siguientes interrogantes:

1. El test de repertorios básicos para el aprendizaje de las matemáticas ¿Cumple con su propósito de medición?

2. El test de repertorios básicos para el aprendizaje de las matemáticas ¿Presenta una adecuada confiabilidad?

3. Las operaciones lógicas de inclusión, seriación, invariación, identificación numérica y clasificación, consideradas como repertorios básicos para el aprendizaje de las matemáticas ¿Influyen sobre el aprendizaje de las matemáticas?

4. ¿Difieren los niveles promedios de los repertorios básicos en función a la edad?

5. ¿Difieren los ni veles promedios de los repertorios básicos en función al sexo?

6. En qué nivel de desarrollo se encuentran los repertorios básicos del aprendizaje de las matemáticas).

Resolviendo las interrogantes planteadas queremos alcanzar los siguientes objetivos:

1. Proporcionar a la comunidad científica un test válido y confiable que permita determinar:

1.1 El nivel de los repertorios básicos que afectan al aprendizaje de las matemáticas.

1.2 El grado de probable éxito o fracaso en el área de las matemáticas

1.3 Medidas preventivas y correctivas en caso de déficit en los repertorios básicos para el aprendizaje de las matemáticas.

2. Se establecerá si la edad y el sexo influyen o no sobre los repertorios básicos.

\section{IMPORTANCIA Y JUSTIFICACIÓN:}

El desarrollo de la presente investigación se justifica en cuanto el conocimiento de los repertorios básicos para el aprendizaje de las matemáticas como prueba de entrada en la acción del proceso enseñanza-aprendizaje permitirá al docente replantear la secuenciación de pasos necesarios para alcanzar los objetivos que el programa educativo exige. De esta manera se verá disminuido el porcentaje de fracaso y la subsecuente frustración que ello conlleva en los estudiantes, puesto que al eliminar las barreras psicológicas mediacionales para el aprendizaje de esta asignatura, el profesor podrá retornar a la secuenciación inicialmente planteada.

Para los psicólogos una herramienta como esta le permitirá diagnosticar los factores activos en el fracaso en el aprendizaje de las matemáticas. y desde la perspectiva de la ciencia psicológica desarrollar programas correctivos y estimulativos de los factores que se encontrasen deficitarios; incluso dará la pauta para desarrollar programas preventivos.

\section{ALCANCES Y LIMITACIONES}

El test de repertorios básicos para el aprendizaje de las matemáticas está desarrollado para púberes y adolescentes entre los 12 y 18 años de edad con estudios de instrucción secundaria. Sin embargo la prueba puede aplicarse a edades por encima de los 18 ya que también es de esperarse que estos repertorios hayan sido alcanzados anteriormente.

Como el test ha sido realizado empíricamente en Lima metropolitana, pese a que los principios básicos son susceptibles de generalización, no lo son las normas que del presente estudio se desprenden. Estas requerirán de la normalización en el interior del país con muestras representativas de cada sector o región.

Es importante señalar que estos repertorios básicos también son alcanzados EN 
ALGÚN NIVEL por las personas sin estudios secundarios, incluyendo a los analfabetos. Sin embargo, por ahora sólo nos ceñimos a la población letrada de Lima.

\section{ESTUDIOS PREVIOS}

Son muchos los trabajos de investigación desarrollados con el tema de las matemáticas y sus dificultades en el proceso de enseñanza aprendizaje, como el de Carpenter y colaboradores en 1980, Mayer R. En 1980, Sternberg en 1977, Bobrow, 1968, Hayes 1981. Mayer, 1983, Nickolson 1974, Loftus y Supps 1972, Riley 1982 Greeno. 1980. Shoenfeld 1979. Brown y Burton, 1978. Enfocan el problema en relación al tipo de entrena-miento recibido y fracaso, por otro lado como el conjunto de operaciones cognitivas que deben ser estudiadas en relación al aprendizaje de las matemáticas y las estrategias ensayadas con el propósito de disminuir el fracaso.

En nuestro medio Luis A. Vicuña Peri 1984 estudió acerca del proceso de encodificación y decodificación del lenguaje en la solución de problemas aritméticos, y en 1985 se investigó sobre el papel que cumplen las operaciones lógicas propuestas por J. Piaget, en un estudio cuasi experimental, comprobando que los sujetos estimulados en los procesos básicos incrementaban su rendimiento escolar de las matemáticas.

\section{REFERENCIAS TEÓRICAS}

La concepción conductista asume a la conducta psicológica como la resultante de la relación estímulo E-R. Para esta posición el sujeto aprende a responder ante un estímulo y se mantiene merced al refuerzo o consecuencia que acompaña contingente mente a la conducta. De esta manera, si el profesor de matemáticas ofrece mediante estimulaciones audiovisuales un procedimiento operacional, el alumno deberá por instigación emitir la conducta esperada, y cada vez que esta se presente será reforzada de inmediato. Esta acción aumentaría la probabilidad de ocurrencia de la conducta ante tales estímulos. Así, el estudiante puede ser entrenado en operaciones aritméticas y mediante un programa de reforzamiento compuesto-conjuntiva podrá realizar en un tiempo determinado un número específico de relaciones. Este resultado es útil y oportuno, pero sólo aparentemente debido a que no se llega a los principios subyacentes de cada operación. como se tuvo la oportunidad de verificar en el Colegio de Aplicación de San Marcos en el Año de 1984, donde mediante un programa de refuerzo positivo logramos que estudiantes del primero al quinto año realicen operaciones aritméticas a razón de 20 sumas, restas, multiplicaciones y divisiones de cuatro a seis cifras, con números naturales, enteros y decimales en un tiempo de diez minutos con un promedio de error de uno y con una desviación standard también de uno, habiendo el grupo iniciado antes del entrenamiento- con un promedio de error de $20 \mathrm{y}$ una desviación standard de $\mathrm{S}$ (de los ejercicios) en un tiempo promedio de 40 minutos y con una desviación standard de 8 (del tiempo). Los resultados demostraron la eficiencia del programa, sin embargo en un segundo momento se sometió a la misma muestra a problemas donde debían razonar el procedimiento y utilizar las operaciones de dominio, anteriormente mencionadas. Se encontró así un promedio de éxito igual a $\mathrm{S}$ y una desviación de 3 , siendo 20 el número de problemas planteados. Por este resultado quedó al descubierto que el primer entrenamiento no se transfiere de manera proactiva positiva, obligándonos a revisar todo el proceso. De dicho análisis se llegó a la conclusión que la adquisición de una destreza puede ocurrir al margen de procesos de análisis. De esta manera el aprendizaje sería mecánico, considerándose suficiente la rígida aplicación de reglas. Fenómeno similar se encontró en la asignatura de lógica, por ello se llegaba a la conclusión siguiente: 
EL CONOCIMIENTO DE LAS REGLAS NO ES SUFICIENTE PARA QUE EL

\section{SUJETO ALCANZASE UNA SOLUCIÓN RAZONADA A UN PROBLEMA NO MECÁNICO O MEMORISTICO.}

Por lo expuesto tenía que tomarse una decisión entre la adquisición de habilidades mecánicas y la estimulación de la capacidad del pensamiento en cada punto de una actividad de enseñanza. Se buscaría integrar esta nueva adquisición al repertorio existente en el alumno, permitiéndole ampliarlos en dirección a nuevos logros y aplicaciones. Ello nos lleva a afirmar que sólo se cumplirá con los objetivos educacionales si el estudiante logra integrar principios y si los aplica a las diversas circunstancias del vivir cotidiano.

Para la postura mediacional (E-O-R) los procesos subyacentes a la conducta observada cobran una vital importancia. Pshonick en el precondicionamiento sensorial demostró que, aún los perros, no respondían sólo a las características del estímulo sino más bien a un proceso de configuración. Por ejemplo, si dos estímulos se presentan simultáneamente en varias oportunidades y luego según el modelo conductista se forma una respuesta condicionada a uno de ellos y no al otro cuando se presenta el estímulo no reforzado el animal también responde. Pschonick y después Brogden asumen que la presentación de los dos estímulos antes del condicionamiento sólo con uno de ellos han formado en el organismo una configuración, llamada también respuesta central (rc). De allí que el refuerzo a uno de los estímulos establecerá un nexo condicionado según lo plantea I.P.Pavlov. Al ocurrir el estímulo reforzado evocará al que fue reforzado (rc) y esta a su vez activará a los estímulos propioceptivos (e) que finalmente dirigen la conducta observable. Mencionamos estos hallazgos en la medida que nos permiten explicar funcional-mente a las conductas psicológicas. para lo cual utilizamos también los aportes de Tolman. para quien el sujeto no aprende una relación $\mathrm{E}$ - R sino más bien una relación $\mathrm{E}$ - E, donde el estímulo -sea interno o externo- no determina la conducta, cumpliendo sólo el papel de señal para que en base al significado adquirido por el sujeto sea quien decide por una $\mathrm{u}$ otra alternativa, tal como lo demostró en su experimento de condicionamiento de lugar. Así mismo utilizamos los aportes de Osgood en lo referente al aprendizaje verbal por los cuales se demuestra que la forma en que los individuos establecen una comunicación ocurre sólo si existe en el interior de ellos el significado del estímulo sonoro que están recibiendo. Por lo expuesto concluimos que el sujeto tiene que DECODIFICAR el mensaje en función al valor de los códigos que ha aprendido previamente. Así, el significado de una palabra no es precisamente la que un diccionario especializado nos muestra, sino el sentido particular que la persona le da en un contexto y un tiempo específicos. Lo mismo ocurrirá si alguien desea expresar verbalmente algo, pudiendo tener la idea o el sentimiento claros, pero fracasando en el esfuerzo de comunicarlo debido a que no posee los códigos verbales - codificables en su grupo - que expresen claramente lo que intenta dar a conocer. De esta manera estamos ubicándonos en el esquema metodológico E - O - R, donde el estímulo como variable independiente solo inicia el proceso, la $\mathrm{R}$ como variable independiente no es una reacción directa al estímulo sino más bien la síntesis del proceso mediador del organismo, llamada también variable interviniente en las que distinguimos el lenguaje y el pensamiento, la personalidad y el ajuste, la motivación y el aprendizaje, la sensación y la percepción, la internalización de las variables sociales y lo fisiológico, lo patológico y el desarrollo. Sobre la base de los dos últimos y las múltiples interacciones con el medio, (desde la familia hasta la sociedad como medio externo y con desde las bases anaátomo-fisiológicas como medio interno) se desarrollarán e integrarán todos los anteriores, definiendo la propiedad molar y única del psiquismo en cada persona. 
Si bien cada proceso puede ser entendido como una unidad molecular, con autonomía y funciones establecidas, debemos considerar que cumple dichas tareas encaminada a que sus funciones se interrelacionen de manera interactiva con las demás del organismo, buscando que este se adapte. Lo que se intenta exponer en este último párrafo es la posibilidad de ver los procesos en su conjunto como elementos integrados de un sistema mayor que vendría a ser el psiquismo. Si aceptamos esta concepción, podríamos tener información del «todo» analizando alguna de sus «partes». El ejemplo también funciona si consideramos que cualquier cambio sustancial en alguno de los procesos psicológicos desembocará necesariamente en un cambio del resto de ellos.

Un ejemplo podemos encontrarlo en el aprendizaje. Cuando un sujeto debe aprender algo relativamente nuevo deberá integrarlo al repertorio ya existente, por tanto en su ausencia, deficiencia o distorsión el sujeto presentará serias dificultades, pudiendo utilizar como alternativa recursos memotécnicos que no garantizan una acomodación real. Ello lo encontramos en individuos que pueden cantar una canción en algún idioma extranjero pero sin conocer significado alguno. Tal vez se sientan emocionalmente muy capaces e incluso reconfortados pero no llegan a reducir su ignorancia al respecto ya que no podrán operar en lo más elemental para llegar al mensaje.

En la concepción operacionalista de Jean Piaget, que asumimos en el presente estudio, se considera que la estructura del psiquismo está dada por la función cognitiva que contiene al pensamiento lógico y a la organización de la realidad. Sin embargo este enfoque, cuidando la totalidad psíquica sostiene que la fuerza que dinamiza al psiquismo es la función de la afectividad y que ambas encuentran su expresión en la función de la representación. El desarrollo de estas funciones es de manera contigua e isomórfica, distinguiéndose fases del desarrollo que van desde lo más primitivo - como es el uso de los mecanismos reflejos congénitos - hasta coronarse con el pensamiento lógico proposicional.

En cada fase del desarrollo existe formas típicas del comportamiento en las que se van construyendo los esquemas de conducta mediante un proceso de adaptación activa con la realidad, cuya resultante llevará al sujeto a interiorizar su experiencia (asimilación) constituyéndose en esquemas de conducta que mediante nuevas interacciones adaptativas irán ampliando los esquemas primarios (acomodación), donde los primeros esquemas de conducta son sensomotores. Sobre esta base el sujeto será cada vez más independiente de la exploración sensorio-motora. En un inicio se irá dando paso al lenguaje e interpretación perceptiva, pero aún con algún apoyo motor. Ello continuará hasta que esta necesidad por el apoyo motor es reemplazada finalmente por el lenguaje y el pensamiento, sin embargo si las estimulaciones no han sido pertinentes el sujeto podrá hacer uso de un lenguaje y pensamiento proposicional pero de un alcance muy concreto.

Al tiempo que el pensamiento lógico se va desarrollando, el individuo va descubriendo ideas generales sobre las cosas, - va llegando a formar conceptos - es decir, logra organizar la realidad. Para ello descubre el concepto de objeto, que involucra la constancia e invariación de la materia, sustancias y energía. Asimismo llega al concepto de espacio, medición y perspectiva, que facilitan el uso de ordenadas y coordenadas en la invariación de las dimensiones y distancias en el espacio.

Gracias al concepto de objeto se contrarrestará la idea de que estos modifican su dimensión cuando se desplazan y/o nos desplazamos en el espacio. En el segundo caso, se alcanza la noción de que la distancia entre dos cuerpos NO VARIA aún cuando entre ellos interfiera un tercero, siempre que los dos cuerpos continúen en reposo.

Asimismo, respecto a la medición, el sujeto deberá fusionar las operaciones lógicas reversibles de iteración con la transitividad de identidad; esto es si $\mathrm{A}=\mathrm{B}, \mathrm{B}=\mathrm{C}, \mathrm{C}=\mathrm{D}, \mathrm{D}=\mathrm{E}$ 
.... entonces, $\mathrm{A}=\mathrm{E}$. Luego, cualquier elemento de un conjunto transitivo de identidad podrá ser elemento o criterio de medida para lo cual se integra, señalando por ello el número de veces en que el criterio es contenido en $\mathrm{A}$.

En la perspectiva, el sujeto estará en condiciones de ponerse en el punto de referencia del otro, lo que implica la reconstrucción mental para determinar cómo se vería o estaría cuando se ha modificado el ángulo de referencia.

Igualmente el sujeto llegará al concepto de tiempo y velocidad descubriendo e integrando en una misma unidad el tiempo físico con el fisiológico, como criterio básico para entender la velocidad como tal y no por su aproximación a un punto meta.

En lo que es materia de la presente investigación el sujeto deberá alcanzar el concepto de número, lo que resulta de la fusión de las operaciones lógicas de la inclusión y la seriación. En la inclusión el sujeto comprenderá que $\mathrm{A}+\mathrm{A}^{\prime}=\mathrm{B}$ como $\mathrm{A}=\mathrm{B}-\mathrm{A}^{\prime}$. Por la seriación alcanzará a entender que en un conjunto de elementos similares en muchas de sus propiedades y características, si difiere en una de ellas, partiendo de este detalle son susceptibles de ordenamiento sucesivos en el tiempo y contiguo en el espacio, tanto con un criterio ascendente o descendente, de forma tal que según el criterio elegido cualquier elemento es al mismo tiempo mayor o menor que cualquiera de los elementos de la serie. Todo esto permite al sujeto llegar a la propiedad numérica de la asimetría.

Bajo esta concepción podemos considerar que llegar al concepto de número va infinitamente más allá de la posibilidad de que un estudiante nos defina que es el número, puesto que lo que importa es que ambos hayan llegado a comprender que la expresión numérica contiene las operaciones lógicas de inclusión o seriación. Siendo así podemos considerar que el aprendizaje de las matemáticas dependerá en gran medida de la capacidad del sujeto para trabajar reversiblemente con estas operaciones lógicas. Aislaremos entonces la inclusión y la seriación como posibles factores básicos para el adecuado aprendizaje de las matemáticas, agregando la operación de clasificación, la que está muy vinculada con la inclusión, pero a su vez nos permite evaluar del sujeto si tiene la capacidad de formar clases con elementos en base a criterios comunes que pueden ir de lo concreto a lo abstracto.

El trabajo numérico que también lo incluimos como factor en cuanto queremos establecer si el sujeto es capaz de leer y escribir expresiones matemáticas como operar con ellos en lo elemental. Finalmente, hemos considerado a la invariación en cuanto demanda del sujeto la capacidad de manejar verbalmente la constancia e invariación de cantidades continuas y colectivas, que significa haber llegado al concepto de objeto, en otras palabras, a la aplicación básica de la imposibilidad de crear o destruir algo. Asimismo la constancia de dimensiones y distancias de los objetos en el espacio hace su aparición con la anterior, así como la integración del tiempo y la velocidad en un sistema único. Todas estas nociones básicas las consideramos como otros factores trascendentales para el aprendizaje de las matemáticas, ya que implican el conocimiento de las propiedades de identidad. Por tanto, si existe o no la posibilidad de estar operando numéricamente al tiempo de estar comprendiendo la razón que fundamenta el ciclo numérico utilizado dentro de un sistema en particular.

De esta manera suponemos que los repertorios básicos para el eficaz aprendizaje de matemáticas son las operaciones lógicas de inclusión, seriación, clasificación, invariación e interpretación numérica. 
HIPÓTESIS: Se sustenta:

H1 Las operaciones lógicas reversibles influyen significativamente en el aprendizaje eficaz de las matemáticas.

H2 La edad cronológica incide significativamente sobre las operaciones lógicas reversibles.

H3 El sexo incide significativamente sobre las operaciones lógicas reversibles.

\section{IDENTIFICACIÓN DE VARIABLES}

\section{Variables Independientes:}

- Repertorios básicos para el aprendizaje de las matemáticas, inclusión, seriación, clasificación, interpretación numérica e invariación.

- Sexo, edad cronológica y grado escolar.

\section{Variable dependiente:}

- Rendimiento escolar en la asignatura de matemáticas.

\section{Variable Interviniente:}

- Procesos del pensamiento y aprendizaje.

\section{Variable Controlada:}

- Estudiantes púberes y adolescentes de Lima Metropolitana.

\section{DEFINICIÓN DE TERMINOS}

1) Repertorios Básicos, son el conjunto de operaciones lógicas que se van adquiriendo como un producto del aprendizaje, que parten de una base anátomo-fisiológica, y que permiten al sujeto la capacidad de generalizar principios y vivencias. Por esto último no existirán en el sujeto como producto de un aprendizaje mecánico, como podría ser el memorizar una serie de números, una historia, etc. Estos repertorios básicos además permitirán el desarrollo y asimilación de los conceptos y leyes matemáticas. Su déficit, por lo tanto, impedirá un adecuado desempeño en esta materia. Es importante señalar que requieren de una estimulación oportuna y adecuada.

Se distinguen los siguientes: la Inclusión, seriación, clasificación, interpretación numérica y la invariación.

2) Inclusión, operación lógica reversible, medida mediante ítemes donde el sujeto deberá en base a caracteres comunes o principios- considerar a elementos como partes del mismo conjunto. $\left(8=\mathrm{A}+\mathrm{A}^{\prime}\right) \mathrm{Y}$ también que $8-\mathrm{A}=\mathrm{A}^{\prime}$.

3) Seriación, operación lógica reversible donde el sujeto debe lograr series ascendentes o descendentes, debiendo considerar a cada elemento como mayor o menor que el elemento que lo antecede o le sigue, según resulte el criterio de orden.

4) Clasificación, operación lógica reversible para lo cual el sujeto deberá utilizar el principio de identidad para formar clases, cuyo nivel de dificultad va de conjuntos constituidos por elementos concretos hasta conjuntos conceptuales.

5) Interpretación Numérica, capacidad que tiene el sujeto para leer y escribir cantidades y expresiones matemáticas con números naturales, enteros, decimales y fraccionarios. Involucra además a la habilidad para desarrollar operaciones elementales de aritmética. 
6) Invariación, nivel conceptual desarrollado por el sujeto que le permite otorgar al objeto la propiedad de constante, siempre que no se agregue ni se substraiga ninguna parte. Asimismo, el uso del principio de que la materia no se crea ni se destruye siendo sólo susceptible de transformación. Implica también el nivel conceptual respecto al espacio, medición y perspectiva en cuanto determina que el sujeto sea capaz de tratar como constantes a las dimensiones y las distancias cuando interviene la variable desplazamiento de los objetos en el espacio.

7) Aprendizaje de las matemáticas, la definimos como el éxito alcanzado que puede lograr el sujeto en la materia de matemáticas, independientemente del año en que lo lleve.

8) Aprendizaje de las matemáticas, proceso por el cual el sujeto alcanza

\section{METODOLOGÍA}

\section{Método:}

La presente investigación es descriptiva, correlacional y comparativa ya que los procedimientos se sistematizan para comprobar la relación existente entre los repertorios básicos y el aprendizaje de las matemáticas. También para determinar el papel que cumple la edad y el sexo sobre los repertorios básicos. Finalmente permite determinar la constancia y precisión de medición del test elaborado

\section{MÉTODO DE MUESTREO}

Siendo el propósito central la elaboración de un test cuya confiabilidad y validez quede demostrada de manera que la obtención de normas se ajuste con un bajo riesgo de error a la población escolar, el procedimiento utilizado corresponde a la determinación necesaria del $\mathrm{n}$ mero de examinados adecuado con el propósito de obtener ítemes cuyo nivel de dificultad oscile entre el 40 y 50\%. Para tal fin utilizamos la siguiente ecuación.

$\mathrm{N}=\mathrm{Z} 2 /(\mathrm{P} 1-\mathrm{P} 2) 2 *(\mathrm{P} 1+\mathrm{P} 2) * 1-(\mathrm{P} 1+\mathrm{P} 2 / 2)$,

donde

$$
\mathrm{N}=1.962 /(0.55-0.50) *(0.55+0.50) * 1-(0.55+0.50 / 2)=767 \text {. }
$$

De esta manera es suficiente una muestra de 767 sujetos para encontrar Índices cuyo nivel de dificultad fluctúe entre el 40 y $50 \%$, con un margen de error probable del 0.05. Sin embargo, como requerimos obtener normas para población escolar de Lima Metropolitana se estimó un tamaño de muestra con un margen de error del 0, 05 de mil estudiantes, que fueron distribuidos de la manera siguiente en función al grado de instrucción, edad cronológica y sexo. 


\section{Cuadro $\mathbf{N}^{\mathbf{0}} 1$}

De la distribución muestral en función al grado escolar, edad cronológica y sexo de estudiantes pertenecientes a centros educativos estatales de Lima metropolitana

\begin{tabular}{|c|c|c|c|c|c|c|c|c|c|c|c|c|}
\hline Grado & \multirow{2}{*}{\multicolumn{2}{|c|}{$\begin{array}{c}\text { 1ro. } \\
12-13.5\end{array}$}} & \multicolumn{2}{|c|}{$2 d o}$. & \multicolumn{2}{|c|}{ 3ro. } & \multicolumn{2}{|c|}{ 4to. } & \multicolumn{2}{|c|}{5 to. } & \multirow{2}{*}{\multicolumn{2}{|c|}{ TOTAL }} \\
\hline $\mathrm{E}: \mathrm{C}$ & & & \multicolumn{2}{|c|}{$13.6-14.5$} & \multicolumn{2}{|c|}{$14.6-15.5$} & \multicolumn{2}{|c|}{$15.6-16.5$} & \multicolumn{2}{|c|}{$16.6-17.11$} & & \\
\hline Sexo & V & M & V & $\mathrm{M}$ & V & $\mathrm{M}$ & V & $\mathrm{M}$ & V & $\mathrm{M}$ & V & $\mathrm{M}$ \\
\hline $\mathrm{N}$ & 102 & 102 & 104 & 100 & 105 & 95 & 103 & 97 & 101 & 99 & 515 & 493 \\
\hline$\%$ & 10.1 & 10.1 & 10.3 & 9.9 & 10.4 & 9.4 & 10.2 & 9.6 & 10 & 10 & 51 & 49 \\
\hline $\begin{array}{l}\text { Total grado } \\
\text { Edad \% }\end{array}$ & \multicolumn{2}{|c|}{$\begin{array}{c}204 \\
20.3\end{array}$} & \multicolumn{2}{|c|}{$\begin{array}{c}204 \\
20.23\end{array}$} & \multicolumn{2}{|c|}{$\begin{array}{c}200 \\
20.23\end{array}$} & \multicolumn{2}{|c|}{$\begin{array}{c}200 \\
19.84\end{array}$} & \multicolumn{2}{|c|}{$\begin{array}{c}200 \\
19.84\end{array}$} & \multicolumn{2}{|c|}{$\begin{array}{l}1008 \\
100\end{array}$} \\
\hline
\end{tabular}

La elección de los participantes fue siguiendo el método aleatorio en aquellos centros educativos en las que se contó con la aceptación y el apoyo de ese plantel, ubicando las aulas que agrupan homogéneamente en base a la edad cronológica.

\section{RECOLECCIÓN DE DATOS:}

El test se aplicó en forma colectiva a razón de 25 examinados por vez, cuidando la libre participación de los examinados. Se observó en ellos durante el examen motivación e interés, acercándose a preguntar al finalizarla prueba sobre algunas preguntas en cuanto a sus respuestas, mostrando curiosidad por saber si estaban en lo correcto en los ítemes de inclusión y seriación pero especialmente en los ítemes de invariación, teniendo algunas veces que dedicarles tiempo de explicación a manera de estimulación.

\section{MÉSTODO ESTADÍSTICO}

Se utilizó el análisis paramétrico descriptivo, enfatizando en el análisis correlacional. Asimismo se utilizó la estadística inferencial. Los estadísticos calculados corresponden a las medidas de tendencia central, al analísis del producto de momentos de Pearson y a la razón crítica de diferencias de medias.

\section{DESCRIPCIÓN DE TEST}

El test se ha titulado "Test de repertorios básicos para el aprendizaje de las matemáticas" cuyas siglas son RBM-87. El test consta de cinco subtest con el siguiente contenido:

Subtest 1 : Seriación (10 ítemes)

Subtest 2 : Inclusión (10 ítemes)

Subtest 3 : Interpretación numérica

(12 ítemes)

Subtest 4 : Clasificación (10 ítemes)

Subtest 5 : Invariación (12 ítemes) 


\section{FORMA DE APLICACIÓN}

El diseño del test es tipo cuestionario ideado para la aplicación colectiva, aún cuando se pueda aplicar en forma individual.

\section{TIEMPO DE APLICACIÓN}

El tiempo promedio registrado en las aplicaciones corresponde a 55' con una Desviación Estándar de 5, donde el 60\% concluye dentro de los 50 minutos, el $35 \%$ dentro de los 60 minutos, quedando sólo un $5 \%$ dentro de los 3 minutos siguientes. Por tanto el tiempo de acción recomendado es de 60 minutos.

\section{TIPO DE PUNTACIÓN}

Los ítemes son dicotómicos. Se abona un punto al éxito y cero al error. Por tanto el mayor puntaje es un índice de mayor repertorio básico en cada uno de los subtest.

\section{CALIFICACIÓN}

Tiene una duración no mayor de 3 minutos utilizando la clave que se reproduce en el apéndice. Luego las operaciones originales son transformadas a puntuaciones equivalentes en la escala W, cuya media aritmética es 100 y su desviación standard el 15 para cada uno de los subtest y puntaje total, para lo cual se utiliza la tabla de conversión de puntuaciones brutas a puntajes equivalentes $\mathrm{W}$ que se reproduce en el apéndice.

\section{LA CONFIABILIDAD}

El grado de constancia y precisión del test ha utilizado el método de las mitades que parte del supuesto que si el instrumento de medición mantiene constante la magnitud del criterio deben encontrarse valores correlacionales por encima del 0,70 que asegura que la correlación de la prueba total utilizando la ecuación profética de Spearman-Brown sea igual o mayor del 0,80 estimando finalmente el Índice de fiabilidad que nos indica el grado de correlación cuando se elimina el error debido al azar.

Además se correlacionará cada subtest con el puntaje total con la finalidad de analizar si cada uno de los subtest es parte significativa del conjunto. De esta manera se podrá afirmar que todos los subtest son o no parte del repertorio básico para el aprendizaje de las matemáticas. Asimismo, será necesario determinar el grado de relación de cada Ítem con la varianza total mediante el análisis de la consistencia interna utilizando la ecuación de Kuder-Richardson.

Estos análisis se efectuarán para cada uno de los subtest y para la prueba total, de forma tal que se pueda determinar si el comportamiento de cada ítem interviene manteniendo una magnitud constante de medida. 


\section{LA VALIDEZ}

La demostración de si el test cumple con su propósito de construcción es tarea de vital importancia en todo instrumento de medición. Por tal razón, en el presente trabajo donde se quiere determinar si los considerados repertorios básicos para el aprendizaje de las matemáticas son efectivamente tales, habrá la necesidad de demostrar si los contenidos evaluados influyen en el rendimiento escolar de las matemáticas. Para lograrlo se sustentará la hipótesis de que si un factor es básico para el aprendizaje de las matemáticas entonces debe encontrarse diferencias significativas entre los sujetos que puntúan alto en el test frente a los que se ubican dentro del $25 \%$ inferior en los calificativos promedios en el curso de matemáticas.

De esta manera si un examinado tiene alta performance en el test de repertorios básicos también debe tener un rendimiento escolar en las matemáticas significativamente mayor al examinado que tiene baja puntuación en el test. De ser así se puede afirmar que el test discrimina al sujeto de alto y bajo rendimiento escolar en matemáticas. Por lo tanto podemos afirmar que los factores medidos son repertorios básicos para el eficiente aprendizaje de las matemáticas.

Por lo expuesto, desde la validez de constructo el test será válido.

Además se correlacionarán los puntajes del test y cada subtest con los calificativos escolares para establecer una estimación de la validez predictiva, de tal manera que podamos estimar la variable $\mathrm{X}$ partiendo de la variable $\mathrm{Y}$ y viceversa.

Por otro lado se efectuará la correlación inter-subtest con el propósito de establecer si cada uno es un factor diferenciado.

Respecto a los calificativos escolares en el curso de matemáticas que los docentes elevan a las direcciones de estudio es importante señalar que no son los mejores indicadores ya que estos promedios no sólo consideran el resultado de los exámenes sino que incluyen intervenciones orales, tareas para la casa, asignaciones y trabajos extracurriculares. Por tal razón no se tomó en cuenta los promedios sino que aprovechando la gentileza de los docentes- se obtuvo los calificativos de los exámenes escritos, que pese a las limitaciones que este tipo de evaluaciones presenta fueron en todo caso un mejor indicador.

Al no encontrar otro indicador que resulte más efectivo por el momento se tomará como variable concurrente los calificativos de los exámenes escritos del segundo y tercer bimestre. No se considera ni el primero ni el cuarto bimestre. El primero porque el material es breve e introductorio y en el cuarto intervienen otras variables como la motivación por aprobar el curso o elevar el promedio.

\section{Análisis de Ítems}

Los ítemes que integran la prueba fueron seleccionados teniendo en cuenta los siguientes criterios:

1. Se redactaron los ítems y se pusieron a prueba en estudiantes con similares condiciones a las de la muestra, con el propósito de descubrir si algún vocablo semánticamente 
desvirtuaba el sentido del ítem. Esta prueba se hizo en forma individual y colectiva y se seleccionaron los ítems que no generaron dificultad de comprensión, teniendo como indicador la coincidencia en el resultado de la aplicación individual con la aplicación colectiva.

2. Se solicitó la opinión de profesores de lógica y matemáticas respecto a los contenidos de cada ítem, apreciaciones que se tomaron en cuenta siempre que arrojaran un ji cuadrado al 0.01. De esta manera, de 74 ítems iniciales se eliminaron 20, sea por que eran reiterativos o porque medían factores diferentes.

3. Aplicada la prueba a una muestra piloto de 720 sujetos se efectuó el análisis en base al índice de dificultad, cuidando que oscilen entre un rango de 0,40 a 0,60. Sin embargo se dejaron algunos ítems considerados como difíciles aún cuando va en desmedro de la confiabilidad, ya que el contenido fue catalogado como importante para propiciar programas estimulativos.

4. Además del índice de dificultad se tuvo en cuenta el análisis mediante la correlación tetracórica, teniendo como criterios que la magnitud de la correlación resulte significativa al 0,05 de riesgo de error. Para la muestra piloto fue suficiente que alcanzara una correlación mínima de 0,21 siempre que tenga dirección positiva.

\section{TIPIFICACIÓN}

Los puntajes brutos fueron convertidos a puntuaciones equivalentes en la escala $\mathrm{W}$, cuya media aritmética es igual a 100 y desviación standard de 15. Ello nos permite analizar a los examinados en 7 categorías de rendimiento. Para este fin se siguió los pasos clásicos: una vez obtenida la media aritmética, la desviación standard y la asimetría de cada subtest y el puntaje total se convirtieron a puntuaciones $\mathrm{Z}$ y con estos valores se transformaron a puntuaciones $\mathrm{W}$ multiplicando cada valor de $\mathrm{Z}$ por 15 y sumándole luego 100. Así se obtuvo el perfil de rendimiento para cada examinado.

También se han obtenido normas percentilares de los puntajes directos de cada subtest y del test total.

\section{CONTRASTACIÓN DE RESULTADOS}

Siendo uno de nuestros objetivos dejar establecido si la edad cronológica y el sexo influyen sobre los niveles de los repertorios promedios de los repertorios básicos para el aprendizaje de las matemáticas, se compararon bajo la razón crítica de diferencia de medias los resultados de cada subtest y test total, trabajando bajo un riesgo de error del 0,05 para ambos casos.

\section{RESULTADOS}

En esta sección veremos los resultados hallados luego del procesamiento estadístico. Comenzaremos con el análisis de la con fiabilidad, seguido por el análisis de validez, luego 
el análisis de ítems, la tipificación y finalmente la contestación en función a la edad y el sexo.

\section{LA CONFIABILIDAD}

Utilizando el método de las mitades, cada subtest se dividió en ítems nones y pares. Luego se obtuvo la correlación para cada uno de los subtest y puntaje total utilizando el análisis de los productos de momentos de Pearson, cuyos resultados se reproducen en el siguiente cuadro. Mediante la estimación de la confiabilidad con la ecuación profética de Spearman-Brown se encontró coeficientes que van desde un mínimo de 0,81 hasta 0,94. Como se puede observar, la intensidad de la correlación va de alta a muy alta, que son indicadores de constancia y precisión del test.

Tabla $\mathbf{N}^{0} 2$

De coeficientes de correlación por el método de las mitadas para cada subtest y test total

\begin{tabular}{|l|c|c|c|c|c|c|}
\cline { 2 - 7 } \multicolumn{1}{l|}{} & Seriación & Inclusión & Int.Numerica & Clasificación & Invariación & Total \\
\hline r mitades & 0.68 & 0.69 & 0.74 & 0.73 & 0.88 & 0.75 \\
R.Spearman & & & & & & \\
Brown & 0.81 & 0.82 & 0.85 & 0.84 & 0.94 & 0.86 \\
rxo & 0.90 & 0.91 & 0.92 & 0.92 & 0.97 & 0.93 \\
\hline
\end{tabular}

Asimismo se ha estimado la confiabilidad mediante la consistencia interna utilizando la ecuación $\mathrm{R}_{2} \mathrm{O}$ de Kuder Richardson. Para tal propósito se clasificó a los examinado en base a los puntajes obtenidos en el cuartil 3 (Q3) o categoría superior y examinados comprendidos dentro del cuartil $1\left(\mathrm{Q}_{1}\right)$ o inferior, hecho que se sustenta en que los sujetos ubicados en la categoría de superior deben ser quienes contestan correctamente a los ítemes, esperando lo contrario en el grupo inferior. De esta manera la varianza de cada ítem debe ser la máxima, puesto que si la mitad superior contesta correctamente, la mitad inferior IDEALMENTE debe contestar de manera errónea. De ser así la media de cada ítem debe ser 0.5 que re presenta el porcentaje de aciertos al ítem. Por lo tanto, el porcentaje de fracasos debe ser igual a 0,5 y siendo la varianza de un ítem dicotómico el producto de la proporción de éxitos por la proporción de fracasos se obtendría la varianza máxima igual a 0,25 donde las varianzas aumentarán o disminuirán en la medida que la proporción de éxitos sea mayor o menor al 0,5. En el primer caso hablaremos de ítems fáciles ya que el grupo de rendimiento inferior también contesta correctamente al ítem. En el segundo caso el ítem será difícil ya que hay proporción de sujetos de alto rendimiento que han contestado incorrectamente al ítem. Desde este principio si las varianzas son uniformes alrededor del 0,5 tendremos un test cuyos ítems tienen aproximadamente una similar magnitud de medida que en los test psicológicos decimos que tiene similar magnitud de dificultad. De esta manera al

correlacionar la varianza de cada Ítem con la varianza total, para cada una de las escalas y para todo el test se encontraron los resultados que a continuación se reproducen. 
Tabla $\mathbf{N}^{0} 3$

De coeficiente de consistencia interna para cada subtest y test total

\begin{tabular}{|c|}
\hline $\begin{array}{lc}\text { SUBTEST } & \text { r20 }\end{array}$ \\
\hline Seriación .................................... 0.86 \\
\hline Inclusión ................................... 0.88 \\
\hline Interpretación numérica .......... 0.90 \\
\hline Clasificación ............................ 0.87 \\
\hline Invariación ................................. 0.90 \\
\hline Test total .................................... 0.90 \\
\hline
\end{tabular}

Como se podrá apreciar el test de repertorios básicos presenta coeficientes altos y muy altos, lo que indica que los ítems del test tienen un comportamiento métricamente consistente.

\section{LA VALIDEZ}

Como ya se dijera, el propósito del test consiste en obtener una medida que permita diferenciar, en base al repertorio básico para el aprendizaje de las matemáticas, la probabilidad de que un estudiante presente o no dificultad en esta materia académica. De conseguir esta posibilidad discriminatoria estaremos en condiciones de afirmar que los repertorios básicos considerados en el presente test actúan como variables influyentes en el rendimiento escolar de las matemáticas. Para este fin en base al rendimiento en el test se separó a dos grupos constituidos por un total de 504 alumnos: 252 comprendidos en el cuartil 3 y los otros pertenecientes al cuartil 1. Por tanto, si efectivamente estos repertorios son influyentes debemos encontrar diferencias significativas en el rendimiento escolar de las matemáticas. Al proceder la contrastación mediante la razón crítica de diferencia de medias se encontró que los estudiantes de baja performance en el test de repertorios básicos difieren significativamente al 0,01 de riesgo de error de sus compañeros con alta performance en el test de repertorios básicos, razón por la cual se rechaza la posibilidad de que las diferencias encontradas sean producto del azar. Así, se está en condiciones de afirmar que los factores medidos son variables influyentes sobre el rendimiento escolar de las matemáticas, cumpliendo de esta manera el test con su propósito al diferenciar significativamente a los sujetos de alto y bajo rendimiento en el test de repertorios básicos. tal como se podrá ver en el siguiente cuadro. 


\section{Tabla $\mathbf{N}^{\circ} 4$}

De diferencia de medias de rendimiento escolar en la asignatura de matemáticas en función al bajo y alto rendimiento académico en cada subtest y test total de repertorios básicos

\begin{tabular}{|c|c|c|c|c|c|c|c|c|c|c|c|c|}
\hline & \multicolumn{2}{|c|}{ Seriación } & \multicolumn{2}{|c|}{ Inclusión } & \multicolumn{2}{|c|}{ Int.Numér. } & \multicolumn{2}{|c|}{ Clasific. } & \multicolumn{2}{|c|}{ Invariac. } & \multicolumn{2}{|c|}{ TOTAL } \\
\hline & BR & $\mathrm{AR}$ & BR & AR & $\mathrm{BR}$ & AR & BR & AR & BR & AR & BR & $\mathrm{AR}$ \\
\hline $\mathrm{M}$ & 51 & 51 & 51 & 51 & 50 & 50 & 50 & 50 & 50 & 50 & 50 & 50 \\
\hline$\%$ & 10.58 & 13.25 & 9.75 & 15.08 & 6.5 & 13.25 & 10.33 & 12.66 & 10.67 & 13.17 & 9.93 & 13.48 \\
\hline DS & 1.08 & 1.66 & 1.91 & 2.46 & 1.24 & 4.18 & 0.98 & 1.77 & 0.77 & 1.99 & 3.41 & 2.63 \\
\hline $\mathrm{DS}^{2}$ & 1.17 & 2.75 & 3.66 & 6.08 & 1.54 & 17.47 & 0.97 & 3.15 & 0.61 & 3.97 & 11.62 & 6.92 \\
\hline Dif & \multicolumn{2}{|c|}{2.67} & \multicolumn{2}{|c|}{5.33} & \multicolumn{2}{|c|}{6.75} & \multicolumn{2}{|c|}{2.33} & \multicolumn{2}{|c|}{2.5} & \multicolumn{2}{|c|}{3.55} \\
\hline GD & \multicolumn{2}{|c|}{0.67} & \multicolumn{2}{|c|}{0.44} & \multicolumn{2}{|c|}{0.62} & \multirow{2}{*}{\multicolumn{2}{|c|}{$\begin{array}{c}0.29 \\
10.89\end{array}$}} & \multirow{2}{*}{\multicolumn{2}{|c|}{$\begin{array}{c}0.3 \\
8.33\end{array}$}} & \multirow{2}{*}{\multicolumn{2}{|c|}{$\begin{array}{l}0.55 \\
6.45\end{array}$}} \\
\hline $\mathrm{t}$ & \multicolumn{2}{|c|}{9.28} & \multicolumn{2}{|c|}{12.11} & \multicolumn{2}{|c|}{10.89} & & & & & & \\
\hline
\end{tabular}

t' al 0.01 para 8 y 100 g.l. prueba de una cola $=2.64$

Otra estimación que se efectuó fue la Validez Predictiva del Test de Repertorios Básicos con el Rendimiento Escolar de las Matemáticas, para lo cual se obtuvo la correlación partiendo de los repertorios básicos como variable independiente y de rendimiento de matemáticas como variable dependiente y el mismo análisis a la inversa, para lo cual se utilizó el coeficiente de correlación eta. El resultado se encuentra en el cuadro que a continuación se reproduce.

Tabla $\mathbf{N}^{0} 5$

De coeficientes de correlación entre cada subtest, test total, con el rendimiento escolar en la asignatura de matemáticas

\begin{tabular}{|c|c|c|c|c|c|c|}
\hline & Seriación & Inclusión & Int. Num. & Clasific. & Invariac. & TOTAL \\
\hline $\begin{array}{c}\text { rxy } \\
\text { x.Rep.Básic } \\
\text { y.Ren.Escol }\end{array}$ & 0.42 & 0.40 & 0.31 & 0.57 & 0.43 & 0.60 \\
\hline $\mathbf{t}$ & 5.54 & 4.74 & 3.54 & 9.17 & 5.17 & 8.15 \\
\hline $\begin{array}{c}\text { rxy } \\
\text { x.Rep.Básic } \\
\text { y.Ren.Escol }\end{array}$ & 0.27 & 0.28 & 0.21 & 0.53 & 0.42 & 0.51 \\
\hline $\mathbf{t}$ & 3.05 & 3.17 & 2.33 & 6.79 & 5.03 & 6.44 \\
\hline
\end{tabular}


De la lectura del cuadro se desprende que todas las correlaciones son significativas aún al 0,01 del riesgo de menor, lo que deja entrever que el test permite conocer el probable resultado del rendimiento de la asignatura de matemáticas, partiendo del hecho que las correlaciones halladas son verdaderas en un 99\%, existiendo sólo una probabilidad de error por cada 100 datos. Se concluye que el test cumple con su propósito predictivo, aún cuando los Índices de predictividad no sean muy altos, posiblemente debido a que la estimación del rendimiento académico de las matemáticas no es la óptima.

Al efectuar la validez empírica correlacionando cada subtest con el puntaje total y las correlaciones inter-subtest se encontró:

1. En las correlaciones intertest los coeficientes van desde un mínimo de 0,26 hasta un máximo de 0,50 mostrándonos correlaciones moderadas y al mismo tiempo que evalúan factores con un nivel significativo de independencia.

2. En las correlaciones subtets puntaje total, los coeficientes van desde un mínimo de 0.60 hasta un máximo de 0,76 . Todas muy significativas aún para el 0.01 de margen de error, lo que indica que cada uno de los subtest son parte de las operaciones básicas para el aprendizaje de las matemáticas. Estos resultados puede verse en la siguiente tabla.

Tabla $\mathbf{N}^{\circ} 6$

De coeficientes de correlación y test total

\begin{tabular}{|c|c|c|c|c|c|c|}
\hline & Seriación & Inclusión & Int. Núm. & Clasific. & Invariac. & Total \\
\hline $\begin{array}{l}\text { Seriación } \\
\text { Inclusión } \\
\text { Int. Núm } \\
\text { Clasific. } \\
\text { Invariac. } \\
\text { Total }\end{array}$ & 1 & $\begin{array}{c}0.28 \\
1\end{array}$ & $\begin{array}{c}0.36 \\
0.46 \\
1\end{array}$ & $\begin{array}{c}0.32 \\
0.40 \\
0.38 \\
1\end{array}$ & $\begin{array}{c}0.29 \\
0.45 \\
0.37 \\
0.39 \\
1\end{array}$ & $\begin{array}{c}0 . .60 \\
0.74 \\
0.76 \\
0.68 \\
0.73 \\
1\end{array}$ \\
\hline
\end{tabular}

\section{ANALISIS FACTORIAL}

Con el propósito de identificar si las variables: seriación, inclusión, interpretación numéricas e invariación son componentes de un factor único se precedió en primer lugar a calcular la determinante de la matriz de correlaciones inter test respecto al modelo hipotético que supone correlaciones de cero a uno, cuyo resultado de la determinante de correlación de la matriz es igual a 0.3726805 que con el test de Bartlett de esfericidad arroja un valor igual a 999.47540 cuyo nivel de significación es 0,00 y siendo el valor determinante de la matriz igual a 0.37 es muy significativa quedando justificado el análisis factorial.

Demostrado que las correlaciones intertest del modelo son significativos se precedió a la factorización mediante el análisis principales componentes obteniendo los pesos totales (Eigenvalues) y pesos relativos (\% de varianza) para cada factor hipotético para nuestro modelo de cinco variables, siendo los resultados los que a continuación se reproducen:

De lo hallado según el SECREE test de Cattel con el programa de Kaiser se aceptan los factores con Eigenvalue mayor o igual a 1 que en el presente caso de los cinco posibles factores se demuestra que solo existe uno. Por tanto, las cinco variables pertenecen a un 
solo factor, es decir, a las operaciones básicas para las matemáticas. Esos resultados se grafican a continuación.

Tabla No. 7

De análisis de principales componentes

\begin{tabular}{|c|c|c|c|}
\hline Factor & Eigenvaluc & $\begin{array}{c}\text { \% de } \\
\text { Varianz }\end{array}$ & $\begin{array}{r}\text { \% Acuml. } \\
\text { de Varian. }\end{array}$ \\
\hline 1 & 2.47803 & 49.6 & 49.6 \\
2 & 0.78487 & 15.7 & 63.3 \\
3 & 0.63601 & 12.7 & 78.0 \\
4 & 0.59344 & 11.9 & 89.8 \\
5 & 0.50754 & 10.2 & 100.0 \\
\hline
\end{tabular}

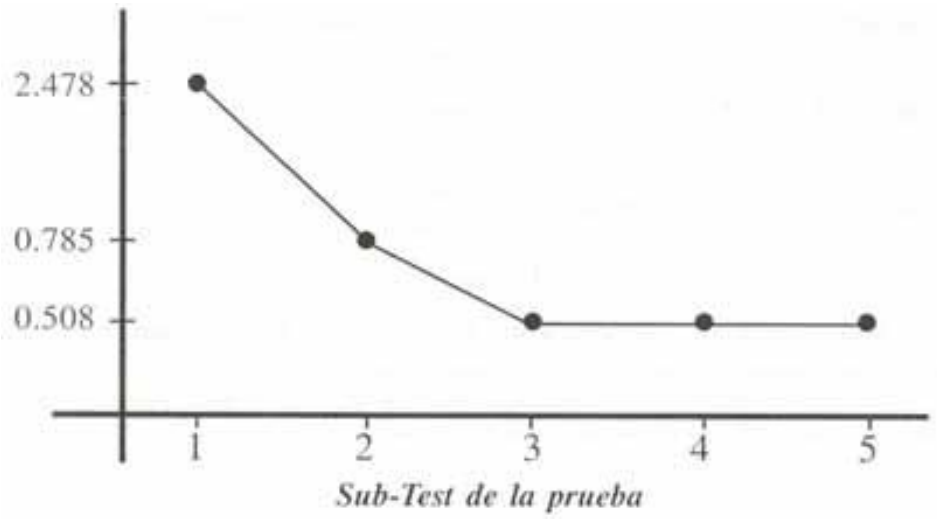

Se observa que solo existe un factor, cuyo peso relativo de cada una de las variables con dicho factor es el siguiente:

Tabla $\mathbf{N}^{\circ} 8$

Peso relativo de cada variable con el factor

\begin{tabular}{|c|c|}
\hline & Factor 1 \\
\hline Seriación & 0.60069 \\
Inclusión & 0.75163 \\
Int. Numérica & 0.73971 \\
Clasificación & 0.71032 \\
Invariación & 0.70718 \\
\hline
\end{tabular}

Por lo tanto, no es posible rotar, quedando demostrada la presencia de un solo factor, quedando en concordancia con el modelo del presente estudio.

Así, la totalidad del test mide las operaciones básicas para las matemáticas.

No obstante hemos creído necesario efectuar el análisis factorial confirmatorio con el programa EQS, partiendo del modelo escrito en la siguiente ecuación:
V1 Seriacion : F1 + E1;
V2 Inclusion : F1 + E2;
V3 Interpret. Numer. : F1 + E3;
V4 Clasificasion : F1 + E4;
V5 Invariacion : F1 + E5; 
Hemos partido del supuesto que el modelo por hallarse sea igual al modelo teórico, esto es, de un solo factor, para lo cual se ha aplicado el test de bondad de ajuste y el Índice de adecuación, encontrándose los siguientes resultados:

Tabla $\mathbf{N}^{\circ} 9$

Test de bondad de ajuste y del índice de adecuación

\begin{tabular}{|c|c|}
\hline \multicolumn{2}{|c|}{ Modelo de independencia Chi cuadrada: 97.798 para 10 g.l. } \\
\hline Independencia AIC : 77.79816 & Independencia AIC : 41.74656 \\
\hline Modelo AIC $\quad:-13.25492$ & Modelo AIC $\quad: 45.70145$ \\
\hline \multicolumn{2}{|c|}{ Chi Cuadrado : 4.745 para 9 g.l } \\
\hline $\begin{array}{l}\text { Valor de la probabilidad para Chi cuadrado } \\
\text { La chi cuadrado normal teórica RLS nara este modelo }\end{array}$ & $: 0.85594$ \\
\hline \multicolumn{2}{|c|}{ Índice de Adecuación de los resultados } \\
\hline Índice de adecuación normalizado Bertlet-Bonnet & $: 0.951($ debe ser mayor o = a 1$)$ \\
\hline Índice de adecuación No normalizado Bertlet-Bonnet & $0.054($ debe ser mayor o $=\mathrm{a} 1.10)$ \\
\hline Índice comparativo de adecuación & $: 1.000($ debe ser menor $\mathrm{o}=\mathrm{a} 1)$ \\
\hline
\end{tabular}

Por la bondad de ajuste, al someter a la prueba del chi cuadrado como criterio de independencia se confirma que el modelo factorial hallado es igual a un solo factor, ratificado por los Índices de adecuación, lo que significa que la solución estandarizada de los pesos de cada variable al factor único van desde un mínimo de 0,579 hasta un máximo de 0,628 y los Índices de fiabilidad de cada variable dentro del factor hallado van de un mínimo de 0,778 hasta un máximo de 0,815 , valores que pueden verse en la siguiente tabla.

\section{Solución estandarizada de la ecuación del modelo confirmatorio, pesos de cada variable al factor único y fiabilidad de variable}

\begin{tabular}{|l|l|l|l|}
\hline Seriación & $\mathrm{V} 1$ & $0.579 \mathrm{~F} 1+0.815$ & $\mathrm{E} 1$ \\
Inclusión & $\mathrm{V} 2$ & $0.628 \mathrm{~F} 1+0.778$ & $\mathrm{E} 2$ \\
Interpretac. & $\mathrm{V} 3$ & $0.623 \mathrm{~F} 1+0.782$ & $\mathrm{E} 3$ \\
Clasificación & $\mathrm{V} 4$ & $0.612 \mathrm{~F} 1+0.791$ & $\mathrm{E} 4$ \\
Invariación & $\mathrm{V} 5$ & $0.611 \mathrm{~F} 1+0.791$ & $\mathrm{E} 5$ \\
\hline
\end{tabular}

Siendo el mínimo de cada peso para resultar significativo aún para el 0,01de riesgo de error pesos hasta OJO y los índices de fiabilidad adecuadas de cada variable igualo mayor de 0,70 se concluye que el test elaborado mide un sólo factor, donde cada subtest o variables mantienen su relativa independencia pero al mismo tiempo son componentes del modelo teórico asumido como operaciones básicas para las matemáticas, quedando confirmado que según el constructo asumido en el test, este cumple con su propósito. 


\section{ANÁLISIS DE ITEMS:}

Los Ítemes de la prueba final han sido seleccionados en base al Índice de dificultad, a la dirección e intensidad de la correlación tetracórica, teniendo en cuenta en la selección de cada Ítem que el Índice de dificultad de preferencia esté dado en el rango 0,40 a 0,60. La dirección de la correlación positiva y la intensidad que resulte significativa que para la

\begin{tabular}{|c|c|c|c|c|c|}
\hline them & ธA.G.A.R & FA.G.S.R & $\begin{array}{l}\text { Poder } \\
\text { Dis. }\end{array}$ & $n$ & Rate? \\
\hline 1 & 0.75 & 0.48 & 0,62 & 0.58 & Si \\
\hline 2 & 0.48 & 0.23 & 0.36 & 0.56 & Si \\
\hline 3 & 0.80 & 0.82 & 0.51 & 0.80 & Si \\
\hline 4 & 0.40 & 0.12 & 0.31 & 0.56 & Si \\
\hline 5 & 0.27 & 0.13 & 0.30 & 0.56 & St \\
\hline 6 & 0.65 & 0,21 & 0.43 & 0.69 & Si \\
\hline 7 & 0.53 & 0,27 & 0,40 & 0.62 & Si \\
\hline 8 & 0.50 & 0,10 & 0.30 & 0,70 & Si \\
\hline 9 & 0.75 & 0.17 & 0,46 & 0.89 & Si \\
\hline 10 & 0,72 & 0.28 & 0.50 & 0.56 & St \\
\hline 11 & 0.55 & 0.28 & 0.36 & 0.56 & Si \\
\hline 12 & 0.59 & 0,27 & 0,38 & 0.56 & Si \\
\hline 13 & 0.67 & 0.23 & 0.45 & 0.58 & St \\
\hline 14 & 0.52 & 0.20 & 0.36 & 0.56 & St \\
\hline 15 & 0.50 & 0.15 & 0.33 & 0.60 & $3 t$ \\
\hline 16 & $0.8 \mathrm{x}$ & 0.38 & 0.63 & $\begin{array}{l}0.76 \\
0.68\end{array}$ & $\frac{S t}{8 t}$ \\
\hline 17 & 0.76 & 0.30 & 0.53 & $\begin{array}{l}0.68 \\
0.68\end{array}$ & $\begin{array}{l}3 t \\
\text { st }\end{array}$ \\
\hline 18 & $\begin{array}{l}0.53 \\
0.57\end{array}$ & 0.15 & 0.35 & $\begin{array}{l}0.68 \\
0.72\end{array}$ & $\sqrt[5 x]{56}$ \\
\hline $\begin{array}{l}19 \\
20\end{array}$ & $\begin{array}{l}0.82 \\
0.60\end{array}$ & $\begin{array}{l}0.32 \\
0.22\end{array}$ & $\begin{array}{l}0.37 \\
0.41\end{array}$ & 0.72 & Si \\
\hline 21 & 0.55 & 0.15 & 0.35 & 0.56 & $\mathrm{Si}$ \\
\hline 22 & 0,67 & 0.22 & 0.50 & 0.78 & 5 \\
\hline 23 & 0.92 & 0.45 & 0.68 & 0.80 & Si \\
\hline 24 & 0.73 & 0.28 & 0.51 & 0.68 & Sit \\
\hline 25 & 0.54 & 0.16 & 0.38 & 0.56 & Si \\
\hline 26 & 0.78 & 0.30 & 0.54 & 0.70 & 5 \\
\hline 27 & 0.82 & 0.35 & 0.58 & 0.78 & 5 \\
\hline 28 & 0.57 & 0.15 & 0.36 & 0.66 & St \\
\hline 29 & 0.48 & 0,15 & $0.3 !$ & 0.56 & So \\
\hline 30 & 0.78 & 0.23 & 0.51 & $\begin{array}{l}0.76 \\
0.6\end{array}$ & ${ }_{5}$ \\
\hline 31 & 0.70 & 0.27 & 0.48 & $\begin{array}{l}0.62 \\
0.56\end{array}$ & Sis \\
\hline$\frac{32}{12}$ & 0.57 & 0.25 & 0.41 & $\begin{array}{l}0,56 \\
0.68\end{array}$ & se \\
\hline 34 & $\begin{array}{l}0.30 \\
0.40\end{array}$ & $\begin{array}{l}0.12 \\
0.17\end{array}$ & $\begin{array}{l}0.31 \\
0.32\end{array}$ & $\begin{array}{l}0.08 \\
0.58\end{array}$ & Si \\
\hline 35 & 0.87 . & 0.42 & 0.63 & 0.72 & $\mathrm{Sa}$ \\
\hline 36 & 0.60 & 0.17 & 0.39 & 0,64 & 5 \\
\hline 37 & 0.58 & 0.27 & 0.82 & 0,56 & Si \\
\hline 38 & 0.48 & 0.13 & 0.31 & 0.64 & $\$ 8$ \\
\hline 39 & 0.50 & 0.12 & 0.31 & 0,64 & 5 \\
\hline 40 & 0.63 & 0.17 & 0,40 & 0,64 & 51 \\
\hline 41 & 0.50 & 0.15 & 0.33 & 0,60 & $\mathrm{Si}$ \\
\hline 42 & 0.50 & 0.15 & 0.33 & 0,60 & Si \\
\hline 43 & 0.87 & 0.27 & 0.57 & 0,74 & 3 \\
\hline 44 & 0.70 & 0.35 & 0.52 & 0.60 & $\begin{array}{l}\text { SI } \\
\text { S4 }\end{array}$ \\
\hline 45 & 0.78 & 0.12 & 0.45 & $\begin{array}{l}0.88 \\
0.68\end{array}$ & $\begin{array}{l}\mathrm{S} \\
\mathrm{S}\end{array}$ \\
\hline $\begin{array}{l}46 \\
47\end{array}$ & $\begin{array}{l}0.75 \\
0.93\end{array}$ & $\begin{array}{l}0.30 \\
0.40\end{array}$ & $\begin{array}{l}0.52 \\
0.66\end{array}$ & $\begin{array}{l}0,68 \\
0.80\end{array}$ & Si \\
\hline 48 & 0.63 & 0.23 & 0.43 & 0.60 & $\$$ \\
\hline 49 & $0 \leq 9$ & 0.17 & 0.34 & 0.58 & Si \\
\hline 50 & 0.73 & 0.34 & 0.55 & 0.58 & Sid \\
\hline 51 & 0.52 & 0.18 & 0.35 & 0.56 & Si \\
\hline 52 & 0.55 & 0.15 & 0.35 & 0.66 & Si \\
\hline 53 & 0.62 & 0.23 & 0.42 & 0.64 & $\mathrm{Si}$ \\
\hline 54 & 0.50 & 0.15 & 0.33 & 0,58 & Si \\
\hline 55. & 0.73 & 0.34 & 0.55 & 0.58 & 5 \\
\hline
\end{tabular}

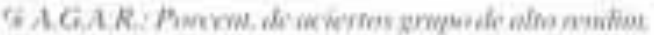


muestra es de 0,21 .

Es importante señalar que algunos Ítemes fueron retenidos aún cuando por el Índice de dificultad se mostraron como difíciles. La justificación se encuentra en la necesidad de mantener el contenido del Ítem por su valor cuantitativo y su implicancia sobre el lenguaje lógico-matemático, para detectarlo y planificar la estimulación correctiva, como es el caso del Ítem $\mathrm{N}^{\circ} 49$.

Para efectuar este análisis la muestra fue dividida en función al cuartil 1 y cuartil 3 teniendo en cuenta el principio de que los ítemes deben mantener una dirección positiva en la correlación, que sólo se consigue cuando el porcentaje de éxito es mayor en el grupo superior. De lo contrario el ítem tiene dirección negativa y automáticamente quedaría fuera del test. También quedaría fuera del test si un Ítem fuera contestado por más del $60 \%$ en ambos grupos. Si se diera el caso el ítem sería de dominio común y por tanto no mostraría utilidad discriminativa entre uno y otro grupo.

Cuando se computaron las respuestas del 50\% de la muestra examinada, es decir, 604 sujetos, siendo el $25 \%$ de rendimiento superior y el otro $25 \%$ de rendimiento inferior en el test de operaciones básicas.

Del análisis de ítems en base al índice de dificultad y correlación tetracórica, con una muestra de 604 estudiantes pertenecientes a colegios estatales dicotomizados en alto y bajo rendimiento en el test de operaciones básicas.

\section{TIPIFICACIÓN}

Los puntajes directos o brutos fueron convertidos a puntuaciones estándar W cuya media aritmética es 100 y desviación estándar es 15 . También se convirtieron dichos puntajes a sus equivalentes percentilares, teniendo en cuenta como cuestión previa el análisis complementario en función al sexo y la edad cronológica, con el propósito de decidir si es necesario obtener normas para cada edad y sexo o de lo contrario, de no encontrarse diferencias significativas en los puntajes directos, optar por normas generales. Para este fin, requerimos el análisis previo del papel que cumple el sexo y la edad sobre los repertorios básicos para el aprendizaje de las matemáticas.

\section{Las operaciones básicas para el aprendizaje de las matemáticas en función al sexo}

Para analizar si el sexo es una variable influyente sobre los repertorios básicos para el aprendizaje de las matemáticas se contrastaron las medias aritméticas dentro de cada edad cronológica en función al sexo para cada uno de los repertorios considerados en el presente estudio y que a continuación se describen.

\section{A) El sexo y el repertorio básico de la seriación.}

Al contrastar 180s medias aritméticas de los cinco grupos de edad que a su vez corresponden del primero al quinto año de secundaria, se ha encontrado diferencias significativas en todos los grupos de edad, siendo los varones quienes presentan promedios significativamente mayores.

\section{B) El sexo y la operación básica de la inclusión}

En un análisis similar al anterior se encuentra que en los cinco grupos de edad el sexo no plantea diferencias significativas, aun cuando las medias aritméticas son mayores en los 
varones respecto a las mujeres. Este dato nos deja entrever que en la operación mencionada, la capacidad para encontrar elementos comunes y extrapolarlos a clases de mayor complejidad que actúan como unificadoras no se ven afectados SIGNIFICATIVAMENTE por el sexo.

\section{C) La operación básica de la Interpretación Numérica y el Sexo}

Al contrastar las medias aritméticas de los varones y mujeres dentro de cada grupo cronológico no se encuentra diferencias significativas. Al igual que en la operación básica de la inclusión los varones presentan medias aritméticas mayores que las obtenidas por el grupo de las mujeres, sin embargo dicha diferencia no puede ser considerada TRASCENDENTE. Este dato nos muestra la posible influencia de la escolaridad sobre el rendimiento escolar en las matemáticas.

\section{D) La operación básica de la Clasificación y el Sexo}

De la razón crítica de la diferencia de medias mediante la prueba t el sexo plantea diferencias significativas en cada uno de los grupos de edad, siendo los varones quienes registran valores promedios significativamente mayores que el grupo de las mujeres.

\section{E) La operación básica de la Invariación y el Sexo}

La operación básica de la invariación y el sexo. De la contrastación de medias aritméticas nuevamente encontramos que el sexo plantea diferencias significativas en cada uno de los grupos de edad estudiados, siendo también los varones quienes presentan puntajes promedios mayores que las mujeres.

\section{F) La operación básica del Test Total y el Sexo}

Al efectuar las comparaciones de medias aritméticas del promedio de los puntajes totales de cada grupo cronológico se encuentra que los varones presentan puntajes promedios SIGNIFICATIVAMENTE MAYORES que las mujeres.

Por lo hallado se justifica la elaboración de normas independientes en función al sexo, sobre todo en las operaciones básicas de seriación, clasificación, invariación y por ende en el puntaje total. Sin embargo para adoptar una decisión final resulta conveniente efectuar las contrastaciones de los puntajes totales de cada uno de las operaciones básicos y test totales en función al grupo cronológico.

Las operaciones básicas para el aprendizaje de las matemáticas en función a la edad cronológica.

La necesidad de hacer este análisis radica en estudios previos respecto a la inteligencia, aptitudes y habilidades que prueban que tales son sensibles a la edad cronológica; no como condición pasiva en el sentido del siguiente isomorfismo: a mayor edad, mayor capacidad espontánea- sino que tal incremento cronológico involucra un aumento de experiencia, tanto cognitiva como emocional, que permitirá al individuo la adquisición de estrategias, dotándolo de una mayor destreza para enfrentar los problemas de la vida cotidiana y los que se suscitan en el proceso cognitivo académico. Por esta razón, conociendo que el sexo influye sobre tres de las operaciones básicas y sobre el puntaje total del test, se procedió a contrastar los resultados promedios en función a la edad 
cronológica, encontrando lo siguiente:

\section{A. La operación básica de la seriación y la edad cronológica.}

Al efectuar las contrastaciones de las medias aritméticas se encuentra que el grupo de los 1213 años 5 meses no difieren significativamente del grupo de 13 años 6 meses a 14 con 5 meses. Sin embargo los del segundo grupo en cuestión presentan promedios SIGNIFICATIVAMENTE MENORES respecto a los grupos de edad de 14 años 6 meses a 15 años 5 meses. A su vez este último grupo no difiere significativamente de los grupos de edad 15 años 5 meses hasta 17 años 11 meses.

\section{B. Operación básica de inclusión y edad cronológica.}

Al contrastar los promedios mediante la razón crítica de diferencia de medias se encuentran las siguientes diferencias significativas:

* Los sujetos de 13 años 6 meses a 14 años 5 meses presentan un promedio significativamente mayor que los púberes de 12 a 13 años 5 meses.

* Los adolescentes de 14 años 6 meses a 15 años 5 meses registran un promedio significativamente mayor que los púberes de 13 años 6 meses a 14 años 5 meses.

* Los adolescentes de 15 años 5 meses a 16 años 5 meses difieren en su valor promedio de los adolescentes de 14 años 6 meses a 15 años 5 meses.

${ }^{*}$ Los adolescentes de 16 años 6 meses a 17 años 11 meses no difieren de los adolescentes de 15 años 5 meses a 16 años 5 meses.

\section{La operación básica de la Interpretación Numérica y la edad cronológica.}

En este análisis comparativo se encuentra el resultado similar al análisis correspondiente a la operación básica de la seriación, ya que los púberes de 12 hasta los 14 años y cinco meses no difieren, como sí respecto a los adolescentes de 14 años seis meses a 15 años cinco meses. Este último grupo de edad a su vez NO difiere de los dos siguientes, dejando entrever la presencia de dos estadios claramente delimitados.

\section{La operación básica de la clasificación y la edad cronológica.}

Al no registrar diferencias significativas entre los 12 años a 13 con cinco meses con los púberes 13 años y medio a 14 con cinco meses, podemos inferir que este rango cronológico determinan ambos un sólo estadio. La presente hipótesis se confirma al encontrar que los adolescentes de 14 años y medio a 15 con cinco meses registran un promedio significativamente mayor que los dos anteriores y a su vez es significativamente menor que los adolescentes de 15 años 5 meses a 17 años 11 meses. Lo que nos muestra que pasar de la lógica operatoria a la lógica formal no ocurre de manera abrupta sino con la presencia de un estadio de transición que pertenece a la edad 14 años y medio a 15 años cinco meses, hecho que se ratifica con lo hallado en el repertorio básico de inclusión.

\section{E. La operación básica de la In variación y la edad cronológica.}

En el repertorio básico de la invariación encontramos resultados similares a los hallados en la clasificación y en la inclusión, esto es, ausencia de diferencias entre los 12 años a 13 cinco meses con los púberes de 13 años y medio a 14 años cinco meses. Estos a su vez difieren significativamente de los adolescentes de 14 años y medio a 15 años cinco 
meses. Al mismo tiempo presentan promedio menores que el grupo de adolescentes cuyo rango de edad va de los 15 años cinco meses hasta los 17 años 11 meses.

\section{F. El puntaje del test total y la edad cronológica.}

La contrastación de las medias aritméticas ratifica la presencia de un estadio de transición entre la lógica operatoria y la lógica formal, al encontrar ausencia de diferencias entre los dos primeros grupos (12 a 13 años con 5 meses con 13 y medio a 14 años 5 meses) y estos difieren de los sujetos de 14 años y medio a 15 años 5 meses, quienes a su vez presentan promedios significativamente menores que el grupo cuyo rango de edad va de los 15 y medio años hasta los 17 años 11 meses.

Por lo hallado, hay la necesidad de verificar si efectivamente ocurre la transición entre el estadio preoperatorio al estadio formal, para lo cual se han reagrupado las edades cronológicas constituyendo tres grupos, que los denominaremos grupo A, los comprendidos entre los 12 a 14 años 5 meses; grupo B, comprendidos entre los 14 y medio años y 15 años cinco meses; y el grupo C, los comprendidos entre el rango cronológico 15 años 6 meses a 17 años 11 meses, encontrando los siguientes resultados:

* Operación Básica de la Seriación.- El grupo A presenta promedios significativamente menores a los del grupo B. Este a su vez es menor sin llegar a ser significativo al 0,05 para prueba de una cola respecto al grupo $\mathrm{C}$, pero sí lo es al 0.10. Este último hecho de alguna manera confirma la presencia de los tres estadios.

* Operación Básica de la Inclusión.- Se encuentra que el grupo C es significativamente mayor que el grupo B y este es significativamente mayor que el grupo A, aún para un riesgo de error del 0,01 para pruebas de una cola, ratificando la presencia de los tres estadios.

* Interpretación Numérica.- Al efectuar las contrastaciones el grupo C no difiere del grupo B, pero este es significativamente mayor que el grupo A, lo que se interpreta que dos estadios están marcados con la ausencia de un tercero, posiblemente debido a la fuerte incidencia cognitiva-académica.

* Clasificación.- En este repertorio básico el grupo C es significativamente mayor que el grupo B y este es significativamente mayor que el grupo A, aún para el 0,01 de margen de error para pruebas de una cola, hecho que señala la presencia de tres estadios cuando los procesos que participan son menos afectados por la actividad cognitiva académica.

* Invariación.- Las contrastaciones de los puntajes promedios en este repertorio básico arroja diferencias significativas al 0,05 en prueba de una cola entre el grupo y el grupo B, y al 0,0 1 de margen de error el grupo B es significativamente mayor que el grupo A, hecho que, valga la redundancia, ratifica la presencia de tres estadios.

Puntaje Test Total. - Al efectuar las diferencias de medias de los puntajes totales del test el grupo $\mathrm{C}$ es significativamente mayor que el grupo B, aún para el 0,01 de margen de error en pruebas de una cola e igualmente el grupo B es significativamente mayor que el grupo A. Estos resultados pueden verse en la siguiente tabla. 
Tabla $\mathbf{N}^{\circ} 25$

De medidas de tendencia central y diferencia de medias en función al grupo cronológico o estadios de desarrollo en la fase lógico formal, en el test total

\begin{tabular}{|c|c|c|c|}
\hline Edad & 12 a 14.5 & 14.6 a 15.5 & 15.6 a 17.11 \\
\hline Media & 18.68 & 24.18 & 26.22 \\
\hline Md. & 18 & 24 & 25 \\
\hline Mo & 19 & 21 & 24 \\
\hline DS & 5.89 & 7.636 & 7.46 \\
\hline $\mathrm{DS}^{2}$ & 34.67 & 58.2 & 55.71 \\
\hline K & -0.49 & -0.39 & -0.22 \\
\hline $\mathrm{A}_{3}$ & 0.33 & 0.19 & 0.49 \\
\hline $\mathrm{N}$ & 408 & 200 & 400 \\
\hline $\begin{array}{c}\text { Dif } \\
\mathrm{S}_{\mathrm{D}} \\
\mathrm{t}\end{array}$ & \multicolumn{2}{|c|}{$\begin{array}{l}-0.57 \\
0.61 \\
9.34\end{array}$} & $\begin{array}{r}34 \\
75 \\
15 \\
\end{array}$ \\
\hline
\end{tabular}

De lo hallado en los análisis efectuados en función al sexo y a la edad cronológica, donde el sexo ha planteado diferencias significativas en los repertorios básicos de seriación, clasificación, invariación y test total y en todos los casos, la edad cronológica ha planteado incrementos significativos reagrupando en grupos de edad y que a su vez deben ser interpretados como la presencia de tres estadios en el desarrollo de los repertorios básicos. de la pubertad a la adolescencia donde el primer estadio que presenta características de reversibilidad del pensamiento, pero aún con dificultad para procesar la información siguiendo las estrategias de la lógica proposicional, posiblemente aún con requerimientos de apoyos operatorios.

En el segundo estadio el uso del lenguaje aumenta la capacidad proposicional, pero aún no lo suficiente. Este hecho se demuestra en cuanto se logra una mejor performance, pero que es ampliamente superada por el tercer estadio. En este último se puede afirmar que el lenguaje es la principal herramienta para procesar, traducir y lograr estrategias proposicionales en la solución de problemas.

Por lo tanto, llegamos a la conclusión que las normas deberán elaborarse tanto para varones como para mujeres dentro de cada grupo cronológico o estadio de desarrollo.

Interpretación de Resultados una vez convertidos los Puntajes Directos a Puntuaciones W y Percentiles (Pc), utilice la siguiente tabla para la interpretación en Categorías de Rendimiento.

\section{CATEGORIA MUY ALTA}

Los sujetos comprendidos en esta categoría ha llegado a la coronación de la reversibilidad del pensamiento lógico formal y de organización de la realidad en base al dominio conceptual de los principios que rigen a la materia, sustancia y energía; estando en óptimas condiciones de asimilar y acomodar el lenguaje matemático, aprendiendo con relativa facilidad nuevos contenidos de esta ciencia formal.

\section{CATEGORIA ALTA}

Los sujetos comprendidos en esta categoría ha llegado a la coronación de la reversibilidad del pensamiento sin el requerimiento del apoyo concreto, son ca-paces de 
elaborar estrategias hipotéticas proposicionales a nivel verbal aplicados al aprendizaje de las matemáticas.

\section{CATEGORIA PROMEDIO ALTO}

Los sujetos comprendidos en este nivel están próximos a alcanzar el nivel lógico formal; aunque con algunas limitaciones en su desenvolvimiento en el aprendizaje de las matemáticas tiende a ser adecuado.

\section{CATEGORIA PROMEDIO}

Los sujetos que se encuentran en esta categoría están en el nivel intermedio, en el que se enlazan las lógicas formal y pre-operatoria. Su aprendizaje de las matemáticas no encuentran mayor dificultad siempre y cuando los contenidos estén referidos al uso de reglas pre-establecidos, y los sujetos puedan generalizar, aunque no necesariamente las relacionen con otros aprendizajes matemáticos.

\section{CATEGORIA PROMEDIO BAJO}

Los sujetos comprendidos en esta categoría, se distancian levemente de la lógica operatoria; requiriendo del apoyo fáctico para aprender a usar reglas matemáticas, y su pronóstico en esta ciencia formal es ligeramente desfavorable.

\section{CATEGORIA BAJO}

Los sujetos ubicados en esta categoría, se encuentran en la lógica operatoria, por lo tanto, la reversibilidad del pensamiento requiere necesariamente de un apoyo concreto; y en consecuencia el pronóstico del aprendizaje de las matemáticas en la escuela secundaria es muy desfavorable.

\section{CATEGORIA MUY BAJO}

Los sujetos comprendidos en esta categoría se sitúan en la lógica operatoria equivalente a la de un niño de 9 a 10 años; por lo tanto tienen pronóstico reservado para el aprendizaje de las matemáticas de la escuela secundaria.

\section{DISCUSION DE RESULTADOS}

El promedio en el rendimiento escolar en la asignatura de matemáticas a nivel nacional es desaprobatorio a pesar de haber recibido años de entrenamiento. Esta realidad nacional es compartida aún con los países desarrollados, tal como reportó Carpenter y otros en 1980 cuando al evaluar el proceso educativo en EE.UU. encontraron que más del 70\% de estudiantes de las escuelas públicas fracasaban en la solución de cierto tipo de procesos aritméticos, surgiendo la necesidad de investigar lo que ocurre con la capacidad de las matemáticas desde el enfoque del procesamiento de la información.

Este punto de vista contempla cuatro procesos:

* La traducción o decodificación del problema.

* La integración sobre la base de los conocimientos previos

* La planificación para llegar a las estrategias generales de resolución de problemas; y

* La ejecución en la cual el sujeto utiliza algoritmos básicamente de adición y sustracción.

Las investigaciones realizadas con el propósito de encontrar factores explicativos han arrojado en todos los casos que los sujetos pueden diferir en cada uno de los procesos mencionados. Este hecho nos revela que la exploración de la capacidad de las matemáticas 
analizando procesas cognitivos donde uno debe coadyuvar positivamente al siguiente hasta llegar a la solución correcta de los problemas matemáticos no ha tenido aún consecuencias significativamente favorables. Posiblemente porque este problema requiere del análisis de las operaciones que median cada uno de los procesos mencionados, enlazándolos en funciones globales. Esta afirmación se sustenta además en que el abordaje psicométrico se orientó a cuantificar la eficacia para resolver problemas algorítmicos, teniendo como criterios la aptitud y habilidad numérica, asumidas como un factor de la inteligencia. Sin embargo los resultados obtenidos con test de aptitud y habilidad numérica sólo han logrado una estimación muy gruesa de la capacidad matemática, precisamente por la definición operacional utilizada, que, al ser circular, no logra explicar el éxito o el fracaso. Por tal razón hemos creído necesario centrar nuestra atención en la búsqueda de operaciones que activamente sirvan de enlace, tanto en la decodificación instruccional como a la selección de los conocimientos pertinentes a un determinado problema para que la planificación de las estrategias concluya con la lógica del uso del algoritmo apropiado. Estas operaciones se desprenden de los estudios de Jean Piaget respecto a la función cognitiva, tanto del pensamiento lógico como de la organización de la realidad. El pensamiento debe independizarse del concretismo irreversible hacia una reversibilidad que permita al sujeto integrar diversas clases en base a principios comunes sin que pierdan identidad. Asimismo cualquier elemento es relativo. Por tanto el concepto de número fusiona a las operaciones lógicas reversibles de la inclusión con la seriación, pero al mismo tiempo dentro de las condiciones cambiantes el sujeto debe diferenciar principios constantes que el mundo físico le plantea, llegando a la invariación de la materia, energía y sustancia.

Si estas operaciones actúan como enlace debemos esperar que se constituyan en un factor fuerte, que sin perder su independencia participe global mente sobre el aprendizaje de las matemáticas.

El estudio efectuado ha confirmado este último supuesto al encontrar que los sujetos con mayor configuración en las operaciones lógicas de seriación, inclusión, clasificación e invariación numérica presentan rendimientos en la asignatura de las matemáticas significativamente mayores que los estudiantes de menor configuración en estas operaciones, dándole validez al aporte de Jean Piaget.

Por otro lado, el proceso de decodificación respecto a la lectura, escritura y manejo de reglas en algoritmos básicos es otro componente de enlace que se integra a las operaciones mencionadas. Esta afirmación se sustenta al encontrar en el presente estudio que las cinco variables asumidas como operaciones básicas para las matemáticas constituyen un sólo factor demostrado mediante el análisis factorial.

\section{CONCLUSIONES}

1. Al contrastar el rendimiento académico de las matemáticas en función a las operaciones lógicas reversibles se confirma la hipótesis que tales operaciones plantean diferencias significativas en el rendimiento escolar de las matemáticas.

2. Al analizar los resultados alcanzados por los sujetos en función a la edad cronológica de los mismos se hallaron incrementos significativos, no de forma sucesiva sino más bien por bloques cronológicos. En otros términos, podemos decir que si bien la diferencia entre uno y otro año cronológico no es significativa, sí la es cuando al reagruparse constituyen tres estadios. El primero comprendido entre los rangos cronológicos 12 años a 14 años 5 meses (144 - 173 meses): el segundo estadio es para el rango de 14 años 6 meses a 15 años 5 meses (174 $\neg 185$ meses) y el tercer estadio ubicado entre los rangos 15 años 6 meses y 17 años 11 meses (186 - 215 meses). En este tercer estadio se vería coronado el desarrollo 
lógico-formal del pensamiento y la organización de la realidad, por lo que las diferencias que pudieran existir con edades mayores se esperan no significativas.

3. Al contrastar las operaciones lógicas reversibles en función al sexo se halló:

3.1 En la seriación, los varones presentan promedios significativamente mayores a los doce, catorce, quince y dieciséis años, no así hacia los trece años.

3.2 En la operación de la inclusión el sexo no plantea diferencias significativas en el continuo cronológico.

3.3 En la interpretación numérica el sexo no plantea diferencias significativas en el continuo cronológico

3.4 En la operación lógica de clasificación los varones presentan promedios significativamente mayores en todas las edades insertas en el continuo cronológico.

3.5 En la invariación los varones presentan promedios significativamente mayores en todas la edades evaluadas.

3.6 En el puntaje total del test, en todas las edades estudiadas son los varones quienes presentan promedios significativamente mayores respecto a las mujeres.

4. Los factores de seriación, inclusión, interpretación numérica, clasificación e invariación son, sin perder su independencia, parte de un sólo factor, denominado operaciones básicas para las matemáticas.

\section{RECOMENDACIONES}

1. El test de operaciones básicas para las matemáticas hasta los 17 años 11 meses no ha saturado su puntuación, manteniéndose en un valor promedio del puntaje total próximo a la media teórica del test (26 puntos), por lo tanto sería conveniente aplicar -dicho material en edades superiores, incluyendo estudiantes de educación superior.

2. En el proceso de enseñanza escolarizada los psicólogos, en coordinación con los docentes deben proporcionar el perfil de las operaciones básicas como prueba de entrada y las sugerencias técnicas para la estimulación ad-hoc con el propósito de reducir la tasa de fracasos en el rendimiento escolar de las matemáticas.

3. Es conveniente elaborar programas de estimulación estandarizadas con un alto nivel de validez predictiva para lograr el cambio de un estadio inferior en las operaciones básicas para las matemáticas hacia los estadios óptimos para la adecuada asimilación de la enseñanza escolarizada de las matemáticas.

4. Para próximos estudios se recomienda seguir una metodología longitudinal que confirme el tránsito de los estadios hallados en el presente estudio.

5. Asimismo se hace necesario probar la validez y confiabilidad del test en muestras nacionales con el propósito de unificar criterios metodológicos en las líneas de entrada y de salida a nivel nacional.

\section{BIBLIOGRAFIA}

Addkins Wood, D. Elaboración de test. México. Ed. Trillas, 1968

Bastin, G. Los test psicométricos. Buenos Aires. Ed. Kapeluz, 1968

Carpenter, T. P. ; Mosner J. M. ; Rombery T. A; Adition y Sustraction: A cognitive Perspective, Hillselade, N. J. Ed. Cribaum, 1982

Cortada, N. ; Carro, J. Estadística aplicada. Buenos Aires. Ed. Previas, 1978

Crombach, Lee J. Fundamentos de la Exploración Psicológica. Madrid. Ed. Biblioteca 
Nueva, 1972.

Festinger, L. ; Katz, D. Los métodos de investigación en las ciencias sociales. Buenos Aires. Ed. Paidos, 1968.

Fischer, H. Estadística Aplicada a la Psicología. Buenos Aires. Ed. Paidos, 1970

Galtung, J. Teoría y Método de la Investigación Social (2 Tomos). Buenos Aires. Ed. Eudeba, 1966.

Garret, H. Estadística en Psicología y Educación. Buenos Aires. Ed. Paidos, 1968.

Glass, G. ; Stanley, J. Métodos estadísticos aplicados en las ciencias sociales. España. Ed.

Florenti Hall lnternational, 1976

Guilford, J. P. Psychometric Methods; New York. Ed. Mc. Graw Hill. 1954.

Magnuson, D. Teoría de los Tests. México. Ed. Trillas, 1983.

Manning, S. ; Rosentock, E. Elaboración de Escalas de Actitudes y psicofísica clásica. México. Ed. Trillas. 1979

Nunnaly Jum C. Introducción a la medición psicológica. Buenos Aires. Ed. Centro Regional de Ayuda Técnica 1979

Padua. Jorge. Técnicas de Investigación Aplicada a las Ciencias Sociales. México. Ed. Fondo de Cultura Económica 1979.

Piaget, Jean, Psicología de la Inteligencia. Buenos Aires. Edil. Psique, 1955

Piaget, Jean, La Formación del símbolo en el niño. México, Ed. Fondo de Cultura Económica, 1961

Piaget, Jean, De la Construcción de lo real en el Niño. Buenos Aires, Ed. Proteo, 1965

Piaget, Jean, Seis estudios de Psicología. Barcelona. Ed. Seix Barral, 1968

Piaget, Jean, El nacimiento de la Inteligencia. Madrid. Ed.Aguilar, 1969

Piaget, Jean, Inhelder, B. Génesis de las Estructuras Lógicas Elementales. Buenos Aires. Ed.Guadalupe, 1967

Piaget, Jean, Inhelder, B. Psicología del Niño. Madrid. Ed. Morata, 1969

Piaget, Jean, Szminska, A. Génesis del Número en el Niño. Buenos Aires. Ed. Guadalupe, 1967

Resnick, L.B; Ford W.: The Psychology of Mathematics for Instruccion, Hillsdale, N.J. Ed. Cribaum, 1981

Summers. Medición de las Actitudes. México. Edit. Trillas, 1975.

Sternberg, Robert Las Capacidades Humanas. Un enfoque desde el procesamiento de la información. Barcelona. Ed. Labor Universitaria, 1982.

Sieguel, Sidney. Estadística no paramétrica aplicada en las ciencias de la conducta. México. Ed. Trillas, 1978

Tovella, M.N. Análisis de los ítemes en la construcción de instrumentos psicométricos. México. Ed. Trillas, 1978

Wanegenen Von, M. Medición y evaluación del aprendizaje y del maestro. Bs. As. Ed. Paidos. 1968. 